OPEN ACCESS

Edited by:

Ignacio Arechaga,

University of Cantabria, Spain

Reviewed by:

Peter J. Christie,

University of Texas Health Science Center at Houston, United States Jack Christopher Leo, University of Oslo, Norway

${ }^{*}$ Correspondence: Christoph Dehio christoph.dehio@unibas.ch

Specialty section:

This article was submitted to Microbial Physiology and Metabolism, a section of the journal Frontiers in Microbiology

Received: 13 March 2019 Accepted: 11 April 2019 Published: 03 May 2019

Citation:

Wagner A, Tittes $C$ and Dehio $C$ (2019) Versatility of the BID Domain: Conserved Function as Type-IVSecretion-Signal and Secondarily Evolved Effector Functions Within Bartonella-Infected Host Cells. Front. Microbiol. 10:921. doi: 10.3389/fmicb.2019.00921

\section{Versatility of the BID Domain: Conserved Function as Type-IV- Secretion-Signal and Secondarily Evolved Effector Functions Within Bartonella-Infected Host Cells}

\author{
Alexander Wagner, Colin Tittes and Christoph Dehio* \\ Biozentrum, University of Basel, Basel, Switzerland
}

Bartonella spp. are facultative intracellular pathogens that infect a wide range of mammalian hosts including humans. In order to subvert cellular functions and the innate immune response of their hosts, these pathogens utilize a VirB/VirD4 type-IV-secretion (T4S) system to translocate Bartonella effector proteins (Beps) into host cells. Crucial for this process is the Bep intracellular delivery (BID) domain that together with a C-terminal stretch of positively charged residues constitutes a bipartite T4S signal. This function in T4S is evolutionarily conserved with BID domains present in bacterial toxins and relaxases. Strikingly, some BID domains of Beps have evolved secondary functions to modulate host cell and innate immune pathways in favor of Bartonella infection. For instance, BID domains mediate F-actin-dependent bacterial internalization, inhibition of apoptosis, or modulate cell migration. Recently, crystal structures of three BID domains from different Beps have been solved, revealing a conserved fold formed by a four-helix bundle topped with a hook. While the conserved BID domain fold might preserve its genuine role in T4S, the highly variable surfaces characteristic for BID domains may facilitate secondary functions. In this review, we summarize our current knowledge on evolutionary and structural traits as well as functional aspects of the BID domain with regard to T4S and pathogenesis.

Keywords: Type-IV-secretion, VirB/VirD4, Bartonella, $\alpha$-proteobacteria, Bartonella effector proteins, relaxases, BID domain, pathogenesis

\section{INTRODUCTION}

Type-IV-secretion (T4S) systems are multiprotein complexes embedded in the cell envelope of Gram-negative and Gram-positive bacteria and some archaea (Berge et al., 2017). They represent versatile nanomachines that fulfill diverse functions including (1) contact-dependent transfer of DNA (conjugation), (2) contact-dependent transfer of bacterial effector proteins into eukaryotic host cells, (3) contact-dependent toxin delivery into bacterial cells, (4) secretion of DNA into the extracellular milieu, and (5) uptake of DNA from the environment (Waksman, 2019). T4S systems can be, based on their architectural complexity, categorized into 
two classes: T4AS (12 subunits) and T4BS ( $>25$ subunits) systems (Waksman, 2019). Based on the paradigmatic Agrobacterium tumefaciens VirB/VirD4 T4AS system, the subunits are named VirB1-11 and VirD4 (Li and Christie, 2018). VirB2-11 are essential for the assembly of the T4S system machinery and substrate translocation. The membrane-bound ATPase VirD4-also known as the T4S coupling protein (T4CP) - is crucial for the recognition of T4S substrates prior to translocation.

The majority of T4S substrates possess a non-cleavable T4S signal at their C-termini consisting of only a few positively charged or hydrophobic residues (Christie et al., 2014). However, some T4S signals form a larger structural scaffold, as for instance, the globular TSA domain of conjugative relaxase TraI encoded by plasmid R1 (Redzej et al., 2013). Another example constitutes the approximately 100-aa-long BID (Bep intracellular delivery) domain that together with a short positively charged C-terminal tail forms a bipartite T4S signal proposed to interact with the T4CP (Schulein et al., 2005; Stanger et al., 2017). The BID domain is present in a-proteobacterial toxins, relaxases, and Beps (Bartonella effector proteins) (Figure 1A), the latter representing numerous host cell-targeted effectors of pathogenic Bartonella species (Wagner and Dehio, 2019). The vast majority of BID domain-containing T4S substrates are genetically linked to a T4S system that resembles the canonical A. tumefaciens VirB/VirD4 T4S system (Figure 1B). VirD4-T4CPs associated with BID domaincontaining T4S substrates form a monophyletic group among T4CP subtypes, indicating a coevolutionary trajectory to maintain interaction of this sublineage with BID domains (Schulein et al., 2005). Multiple studies with the model pathogen Bartonella henselae (Bhe) showed that Beps are translocated via a VirB/VirD4 T4S system into various host cell types to modulate diverse cellular and innate immune functions allowing Bartonella to spread and establish longlasting hemotrophic infections in its mammalian host (Schulein et al., 2005; Schmid et al., 2006; Truttmann et al., 2011a; Okujava et al., 2014).

Beps are multi-domain proteins. A majority of Beps possess an N-terminal FIC (Filamentation induced by cAMP) domain that confers posttranslational modifications, a central connecting OB (oligonucleotide binding) fold, and a C-terminal BID domain (Engel et al., 2011; Harms et al., 2017b). A previous genome analysis revealed that $70 \%$ of all Beps and the interbacterial toxin VbhT (present on conjugative plasmid pVbh encoding the Vbh (VirB homologous) T4S system in certain Bartonella spp.) display the canonical FIC-OB-BID architecture (Engel et al., 2011). Furthermore, all Bep repertoires present in the Bartonella genus harbor Beps with a derived domain composition, which likely evolved from a single primordial FIC-OB-BID ancestor via repeated gene duplication and diversification events. The diversification of Bep repertoires occurred independently in three distinct Bartonella lineages, resulting in Bep197-234 in B. ancashensis of lineage 1 (L-1), Bep1-10 in lineage 3 (L-3), and BepA-I in the most species-rich lineage 4 (L-4) (Harms et al., 2017). Instead of a FIC domain, the derived Beps possess tandem-repeated tyrosine phosphorylation (pY) motifs that are phosphorylated by endogenous host cell kinases, and/or additional
BID domains (Wagner and Dehio, 2019). While the original function as T4S signal is preserved among C-terminal BID domains, some BID domains have secondarily evolved effector functions within host cells. These functions include inhibition of apoptosis, bacterial uptake via rearrangement of the F-actin cytoskeleton, and modulation of cell migration of infected host cells (Truttmann et al., 2011a; Pulliainen et al., 2012; Okujava et al., 2014).

In this review, we will focus on the evolution and classification of BID domains based on their presence in different T4S substrates. We will furthermore discuss structural and functional aspects of the BID domain with regard to T4S and the subversion of host cell function.

\section{CLASSIFICATION AND STRUCTURAL FEATURES OF BID DOMAINS}

\section{Classification of BID Domains}

BID domains display high sequence variability. Their classification is mainly based on their position within the multi-domain relaxases and Beps (Figure 1A; Stanger et al., 2017). C-terminal BID domains that serve as part of the T4S signal are designated as tBIDx and nonterminal BID domains as BIDx, with " $\mathrm{x}$ " representing a number indicating whether this BID domain is the first, second, third, or fourth BID domain counted from the N-terminus (Figure 1A). In the Beps, tBIDs have been further subclassified into ancestral tBIDs found in the canonical FIC-OB-BID architecture and derived tBIDs of pY- and multi-BID domain-containing Beps (Stanger et al., 2017).

A recent study reported the presence of BID domains fused to toxin domains (PezT and Zeta) of toxin/antitoxin modules (Harms et al., 2017a). These PezT/Zeta-BID proteins are encoded by conjugative plasmids that are prevalent in various a-proteobacterial genera such as Agrobacterium, Chelativorans, Ochrobactrum, and Sinorhizobium. Although it is not clear whether these PezT/Zeta-BID proteins are translocated through a T4S system, their genetic conservation to a virB/virD4-like T4S system locus suggests that these proteins are bona fide T4S substrates (Figure 1B; Harms et al., 2017a; Wagner and Dehio, 2019). Therefore, we extend the classification of BID domains by introducing tBIDa for those present in toxins found in various $\alpha$-proteobacterial species. In a phylogenetic tree, tBIDa domains form a monophyletic group similar to tBID2 domains of a-proteobacterial relaxases, tBID1 domains of Bartonella TraArelaxases/VbhTs, and the ancient and derived tBID domains found in Beps, confirming that the tBIDa domains form a new class of BID domains (Figure 1C, left). Interestingly, tBIDa domains are more closely related to the tBID domains of Beps than to tBID2 domains of a-proteobacterial relaxases, even though PezT/Zeta-BID proteins are encoded adjacent to the latter (Figure 1B). Thus, tBIDa domains are likely not the result of gene duplication and reshuffling events of relaxase tBID1/2 domains as proposed for the tBID1 domain of VbhT that is virtually identical to the tBID1 domains of Bartonella TraA relaxases (Harms et al., 2017a). 


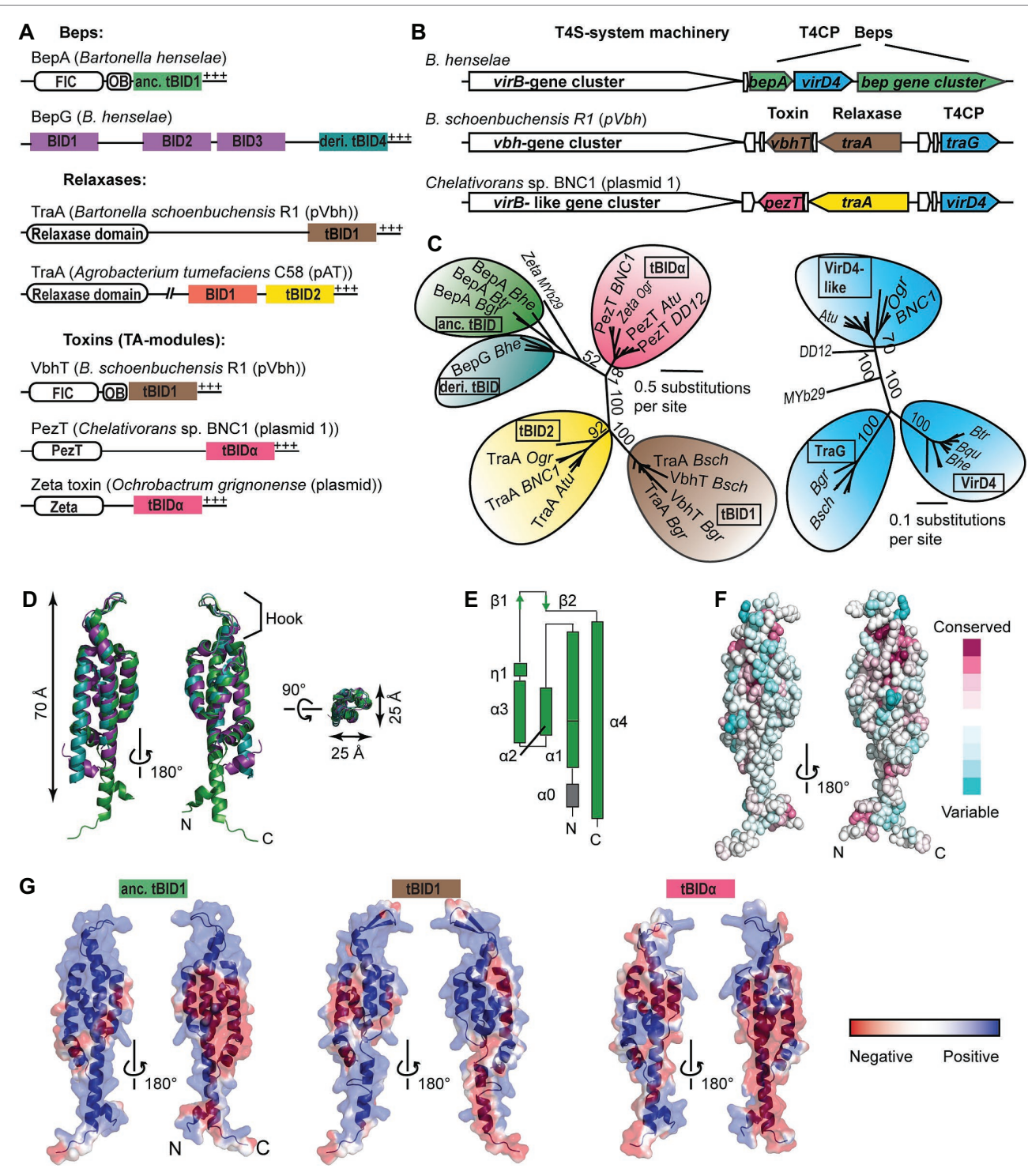

FIGURE 1 | Classification and structural features of BID domains. (A) Domain architecture of BID domain-containing type-IV-secretion (T4S) substrates found within $\alpha$-proteobacteria. N-terminal, catalytic domains (FIC, relaxase domain, PezT, Zeta) and the OB (oligonucleotide binding) fold are displayed as white rectangles, respectively. BIDx and tBIDx domains are colored based on the subclass. +++, positively charged tail; anc., ancestral; deri., derived. (B) Synteny of representative BID domain-containing T4S substrate genes and adjacent virB/virD4-like T4S-system locus and genes involved in plasmid conjugation (the latter two are represented in white). The color code of BID domain-encoding genes is the same as of the respective BID domains displayed in (A). Homologs of T4S coupling protein (T4CP) are depicted in blue. (C) Simplified neighbor-joining distance-based tree representations of the multiple sequence alignment of (left) terminal BIDs [tBIDs, color code as in (A)] and (right) the soluble domains of VirD4-like T4CPs that are associated with the tBIDs. Highlighted are representative proteins (per subfamily) encoded by: B. grahamii (Bgr), B. tribocorum (Btr), B. henselae (Bhe), B. schoenbuchensis pVbh (Bsch), Agrobacterium tumefaciens C58 pAT (Atu), Chelativorans sp. BNC1 plasmid 1 (BNC1), Ochrobactrum grignonense plasmid (Ogr), Ochrobactrum sp. MYb29 (MYb29), and Shinella sp. DD12 (DD12). (D) The similarity and compact nature of the BID fold is highlighted through superposition of the three solved BID domains: BroBep6_tBID1 (green; PDB: 4YK1), Bc/Bep9_tBID1 (cyan; PDB: 4YK2), and BheBepE_BID1 (purple; PDB: 4YK3). (E) Topology representation of BroBep6_tBID1. (D,E) are redrawn from Stanger et al. (2017) to adapt the color code to the remaining figure. (F) Residue conservation is rather low among BID domains of Beps. Depicted is the overall conservation score of BID domains mapped on the surface representation of BroBep6_tBID1. The color code is based on the ConSurf color scale (Ashkenazy et al., 2016). (G) Electrostatic potential mapped on the surfaces of experimentally determined (anc. tBID1 of BroBep6) and modeled (tBID1 of VbhT from Bsch and tBID $\alpha$ from PezT from BNC1) tBID domains. Protein backbones are depicted as cartoon representation. The color code highlighting the electrostatic potential ranges from red (negative) to blue (positive).

The coevolutionary trajectory of tBID domains with their cognate T4CP is evident for tBID1 domains (TraA/VbhT) and TraG and for ancestral/derived tBIDs (Beps) with VirD4 (Figure 1C). In contrast, tBID2 ( $\alpha$-proteobacterial relaxases)- and tBIDa (PezT/Zeta-BID)-containing proteins supposedly interact with the same VirD4-like T4CP (Figure 1C, right), although these tBID domains are as distantly related to each other as tBID1 domains and ancestral/derived tBID domains (Figure 1C, left). It is worth mentioning that translocation of tBID-containing substrates through heterologous VirB/VirD4 T4S systems has 
been experimentally proven in multiple instances (e.g., Schulein et al., 2005; Harms et al., 2017a). For instance, the tBID2 domain of a-proteobacterial relaxase TraA (encoded on Atu pAT) fused to Cre-recombinase (Cre) translocates through the Bhe VirB/VirD4 T4S system with similar efficiency as Cre-tBIDfusions of different Bhe-Beps (Schulein et al., 2005). We therefore believe that coevolution of tBID domains with their BID-associated VirD4-T4CPs has not yet led to the establishment of discrete specificities in recognition - despite the remarkable sequence variability of BID domains.

\section{Structural Features of BID Domains}

Recently, the crystal structures of BID domains representative for three different classes have been solved, including an ancestral tBID1 domain of $B$. rochalimae Bep6, a derived tBID1 domain of $B$. clarridgeiae Bep9, and a derived BID1 domain of Bhe-BepE (Stanger et al., 2017). All three BID domains are folded to a rigid, antiparallel four-helix bundle topped with a hook that accommodates a position opposite to both termini (Figures 1D,E). The shape of the three BID domains is elongated with a length of approximately $70 \AA$ and a diameter of $25 \AA$, suggesting that this conserved fold might be crucial for the primary secretion signal function of the BID domain in T4S (Stanger et al., 2017). It is tempting to speculate that the hook might play a role in the interaction with the T4CP and/or other components of the VirB/VirD4 T4S machinery. Helix-1 ( 1 1) and helix-4 (a4) can either be straight or kinked (Figure 1D). This conformational variability of BID domains at their extremities might be the result of different domain compositions in the remaining part of the protein and/or facilitate novel interactions with host target proteins. Interestingly, the T4S activity of a Cre-tBID1-fusion lacking al was reduced to $30 \%$ compared to full-length Cre-tBID1, suggesting an important role of $\alpha 1$ in translocation (Schulein et al., 2005).

While the hydrophobic core of BID domains is conserved, their surfaces are highly variable. The plasticity of BID domains is highlighted by the fact that BID domains of relaxases and Beps display on average 14\% similarity (Stanger et al., 2017). Sequence similarity is also rather low within the various BID domain classes (Figure 1F), with the highest degree of similarity observed within tBID1 domains of Bartonella TraA and VbhT, respectively. In general, tBIDx subclasses display a higher degree of conservation than BIDx classes, which might be due to a relieved selection pressure of BIDx domains to interact with the T4CP. Although the surface composition among BID domains is poorly conserved, their surface charge distribution seems to be rather consistent, displaying two highly positively charged areas separated by a negatively charged patch (Stanger et al., 2017). This mainly positive charge distribution suggests that BID domains likely interact with a negatively charged surface on the interaction partner. Furthermore, slight differences in the charge distribution between BID domain classes and even among closely related BID domain orthologs can be observed (Figure 1G), suggesting subtle functional differences with respect to T4S efficiency (tBIDx) and effector function (BIDx/tBIDx).
Summarizing, we believe that the conserved rigid fold of the BID domain facilitates its role as T4S signal, whereas the highly variable surface might enable the evolution of secondary functions within host cells.

\section{BID DOMAIN-MEDIATED HOST CELL MODULATIONS}

The remarkable degree of host adaptation of pathogenic Bartonella spp. to their mammalian hosts has been attributed to a large extent to the VirB/VirD4 T4S system and its translocated Beps (Siamer and Dehio, 2015; Dehio and Tsolis, 2017; Wagner and Dehio, 2019). Most of the Bep-mediated host cell modulations are BID domain dependent and include apoptosis inhibition, F-actin rearrangements, and host cell migration. BID domain-mediated phenotypes are best understood on the molecular and cellular level for human pathogenic Bhe and $B$. quintana $(B q u)$. Both species belong to $\mathrm{L}-4$ and thus their Beps are designated with a letter code (BepA, BepB, and so on, Figure 2A).

\section{BepA-tBID1 Mediates Apoptosis Inhibition in a Host-Specific Manner}

Bhe and Bqu enhance the proliferation of human endothelial cells (ECs) by inhibiting apoptosis (Kirby and Nekorchuk, 2002; Schmid et al., 2006; Pulliainen et al., 2012). The antiapoptotic activity was assigned to the ancestral tBID1 domain of Bhe-BepA and Bqu-BepA.2, respectively (Figure 2B). Bhe-BepA-tBID1 physically interacts with the catalytic subunit $\mathrm{C} 2$ of human adenylyl cyclase isoform 7 (AC7) to potentiate cAMP production (Pulliainen et al., 2012). AC7 is a plasma membrane-bound enzyme, which requires activation and association of its two catalytic subunits C1 and C2 to convert ATP into cAMP (Sadana and Dessauer, 2009). Bhe-BepA-tBID1 potentiates the cAMP-triggering effect of GTP-bound $\mathrm{Ga}_{\mathrm{S}}$ in a physiological context and in a pharmacological context together with the plant-derived drug forskolin, which intercalates C1 and C2 into a catalytically active form (Pulliainen et al., 2012). Thus, it is plausible to assume that Bhe-BepA-tBID1 allosterically enhances $\mathrm{C} 1$ and $\mathrm{C} 2$ subunit association in order to elevate cAMP production. The molecular restraints of the Bhe-BepA-tBID1 interaction with C2 (AC7) are currently unknown. However, a Bhe-BepA construct consisting only of helix- 4 of the ancestral tBID1 domain and the adjacent C-terminal stretch did not inhibit apoptosis, suggesting that helix-4 alone is not sufficient for the antiapoptotic activity mediated by Bhe-BepA-BID (Schmid et al., 2006).

Interestingly, neither the BepA paralogs $\mathrm{BepB}$ or $\mathrm{BepC}$ of Bhe nor the BepA ortholog from the rat-associated B. tribocorum (Btr) displayed antiapoptotic activity (Schmid et al., 2006). The latter is in line with previous observations that only L-4 species associated with significant clinical manifestations in humans (e.g., Bhe and Bqu) possess antiapoptotic activity toward human ECs (Kirby and Nekorchuk, 2002). 

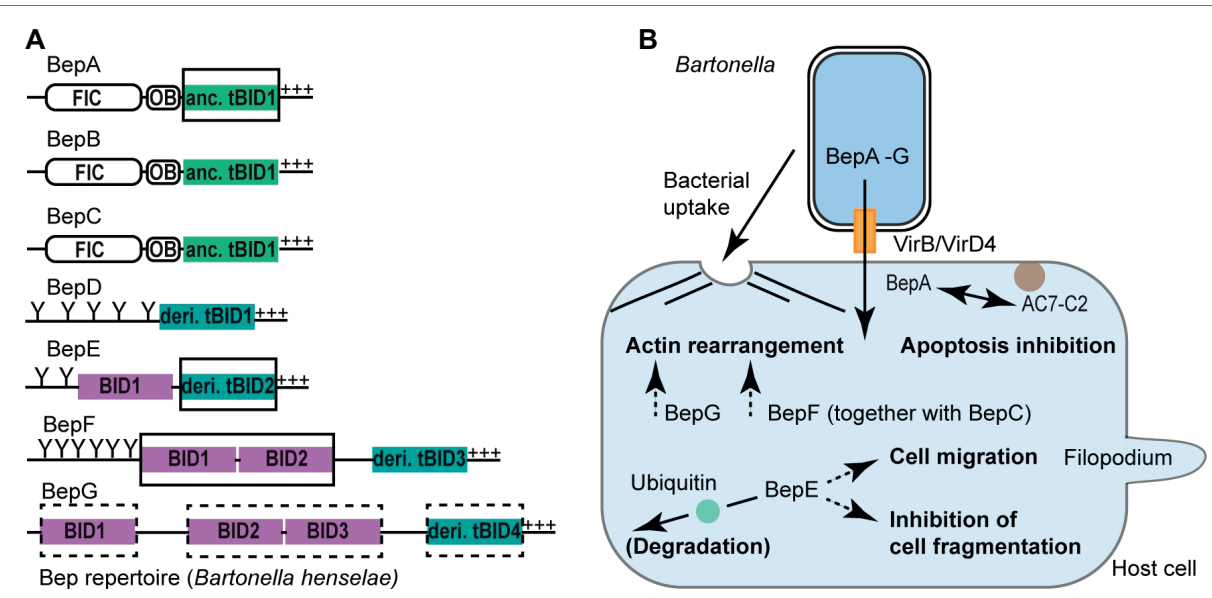

FIGURE 2 | BID domain-mediated host cell modulations. (A) Representative Bep repertoire of the model organism Bartonella henselae highlighting various classes of BID domains. BID domains with experimentally proven or suspected host-modulating function are highlighted with a full or dashed rectangle, respectively. $\mathrm{OB}$, Oligonucleotide binding fold; Y, Tyrosine phosphorylation motif; +++, positively charged tail; anc., ancestral; deri., derived. (B) Schematic representation of BID-mediated host cell modulations. Following the VirB/VirD4 T4S system-mediated translocation into host cells, distinct BID domains [highlighted in (A)] alter host cell signaling processes allowing Bartonella to survive and propagate within the mammalian host. Host cells can counteract Bep-mediated functions by degrading the effectors following ubiquitination of their BID domains as shown for the BID domains of Bqu-BepE. Confirmed BID-target interactions are displayed with a continuous arrow, whereas dashed arrows indicate BID-mediated host cell modulations for which the target protein(s) are currently unknown. AC7-C2, catalytic subunit $\mathrm{C} 2$ of human adenylyl cyclase isoform 7 (AC7).

\section{Multi-BID Domain-Containing Effectors BepG and BepF Trigger Invasome Formation}

Bhe cells are internalized into ECs either individually via endocytosis or as bacterial aggregates in the course of the formation of a unique cellular structure, the invasome (Dehio et al., 1997). Invasome formation is a multistep process, that, following bacterial accumulation at the EC surface, involves F-actin rearrangements and stress fiber formation underneath the engulfed bacterial aggregate (Dehio et al., 1997). Furthermore, in contrast to the T4S system-independent endocytic uptake of Bhe into ECs, invasome formation is strictly VirB/VirD4 dependent (Schmid et al., 2004) and is redundantly triggered by either BepG or through the combined action of BepC and BepF (Figure 2B; Rhomberg et al., 2009; Truttmann et al., 2011b).

BepG of Bhe consists solely of four BID domains that are connected via short linker sequences. Hence, BepG likely promotes invasome formation via at least one of these four BID domains through interaction with yet unknown host target protein(s) (Rhomberg et al., 2009). Similarly, invasome formation mediated by BepF (together with BepC) is triggered by its two nonterminal BID domains BID1 and BID2, but not by its derived tBID3 domain (Truttmann et al., 2011a). Interestingly, BID2 and tBID3 are more similar to each other than to BID1. The overall low sequence conservation of these three BID domains allows no conclusion on why BID1 and BID2 contribute to invasome formation, but tBID3 is negligible in this process. We speculate that, besides certain, non-conserved residues at the surface of BID domains, also the relative position of BID domains within multi-domain architectures may be critical for effector function.

\section{BepE-BID Domains Antagonize Cell Fragmentation and Promote Host Cell Migration}

Bartonella spp. supposedly infect dendritic cells at the dermal site of inoculation and exploit these migratory cells as Trojan horses in order to reach the bloodstream, where bacteria infect, replicate, and persist within erythrocytes (Scherer et al., 1993; Schulein et al., 2001; Okujava et al., 2014; Vieira-Damiani et al., 2016). In vivo dissemination into the bloodstream is VirB/VirD4 dependent and relies on the function of BepE (Figure 2B; Okujava et al., 2014). Bhe-BepE is a pY-containing Bep that contains two BID domains (Figure 2A). Although Bhe-BepE interacts via its pY motifs with several host cell signaling proteins (Selbach et al., 2009), the dissemination of the bacteria into the bloodstream is exclusively dependent on the BID domains. In vitro, the derived tBID2 domain of Bhe-BepE interferes with a deleterious cell fragmentation phenotype triggered by other Beps (Okujava et al., 2014). Inhibition of the cell fragmentation phenotype is not only restricted to Bhe-BepE but appears to be a conserved function among BepE homologs including Bqu-BepE and Btr-BepE (Okujava et al., 2014).

Besides the cytoprotective effect against other Beps, translocated Bhe-BepE promotes the migratory capability of dendritic cells (Okujava et al., 2014). It needs to be determined whether this effect is mediated by the BID domains of BepE or by its pY motifs. However, it is conceivable that the promotion of cell migration is BID dependent, as BID domains of BheBepE are sufficient to enable Bartonella cells to reach the bloodstream in vivo (Okujava et al., 2014).

A recent study showed that the tandemly repeated BID domains of Bqu-BepE but not of Bhe-BepE become ubiquitinated within 
host cells followed by proteasomal degradation (Wang et al., 2018). Whether or not this ubiquitination plays a role in effector function of Bqu-BepE needs to be addressed in future research.

\section{CONCLUDING REMARKS}

The BID domain constitutes together with a positively charged C-terminal tail a T4S-signal that is crucial for the interbacterial transfer of relaxases (alongside plasmid conjugation) and protein toxins by various $\alpha$-proteobacteria and for the interkingdom transfer of effectors by pathogenic Bartonella spp. Besides its genuine role in T4S, several BID domains have adopted secondary functions within host cells such as apoptosis inhibition and F-actin rearrangements. On the basis of the recently solved BID domain structures, their conserved fold may play a crucial role in T4S, whereas the plasticity of the surfaces seems to have facilitated novel interaction areas with host target proteins (Stanger et al., 2017). Future structure/function-related studies should aim to systematically determine key residues of BID domain interactions with the T4CP and/or other T4S system components and with host target proteins. Regarding the latter, comparative analyses of BID domain-host protein interactions evolved in specific Bartonella-mammal pairs will enable us to address the role of Beps in host adaptation.

\section{REFERENCES}

Amyot, W. M., Dejesus, D., and Isberg, R. R. (2013). Poison domains block transit of translocated substrates via the Legionella pneumophila $\mathrm{Icm} / \mathrm{Dot}$ system. Infect. Immun. 81, 3239-3252. doi: 10.1128/iai.00552-13

Ashkenazy, H., Abadi, S., Martz, E., Chay, O., Mayrose, I., Pupko, T., et al. (2016). ConSurf 2016: an improved methodology to estimate and visualize evolutionary conservation in macromolecules. Nucleic Acids Res. 44, W344-W350. doi: 10.1093/nar/gkw408

Berge, C., Waksman, G., and Terradot, L. (2017). Structural and molecular biology of type IV secretion systems. Curr. Top. Microbiol. Immunol. 413, 31-60. doi: 10.1007/978-3-319-75241-9_2

Christie, P. J., Whitaker, N., and Gonzalez-Rivera, C. (2014). Mechanism and structure of the bacterial type IV secretion systems. Biochim. Biophys. Acta 1843, 1578-1591. doi: 10.1016/j.bbamcr.2013.12.019

Dehio, C., Meyer, M., Berger, J., Schwarz, H., and Lanz, C. (1997). Interaction of Bartonella henselae with endothelial cells results in bacterial aggregation on the cell surface and the subsequent engulfment and internalisation of the bacterial aggregate by a unique structure, the invasome. J. Cell Sci. 110, 2141-2154.

Dehio, C., and Tsolis, R. M. (2017). Type IV effector secretion and subversion of host functions by Bartonella and Brucella species. Curr. Top. Microbiol. Immunol. 413, 269-295. doi: 10.1007/978-3-319-75241-9_11

Engel, P., Salzburger, W., Liesch, M., Chang, C. C., Maruyama, S., Lanz, C., et al. (2011). Parallel evolution of a type IV secretion system in radiating lineages of the host-restricted bacterial pathogen Bartonella. PLoS Genet. 7:e1001296. doi: 10.1371/journal.pgen.1001296

Harms, A., Liesch, M., Korner, J., Quebatte, M., Engel, P., and Dehio, C. (2017). A bacterial toxin-antitoxin module is the origin of inter-bacterial and inter-kingdom effectors of Bartonella. PLoS Genet. 13:e1007077. doi: 10.1371/journal.pgen.1007077

Harms, A., Segers, F. H., Quebatte, M., Mistl, C., Manfredi, P., Korner, J., et al. (2017). Evolutionary dynamics of pathoadaptation revealed by three independent acquisitions of the VirB/D4 type IV secretion system in Bartonella. Genome Biol. Evol. 9, 761-776. doi: 10.1093/gbe/evx042
Independent studies showed that $\mathrm{T} 4 \mathrm{~S}$ substrates are translocated to the recipient cytosol in an unfolded state (Amyot et al., 2013; Trokter and Waksman, 2018). Due to its structural similarity with the intramolecular chaperone IpaD of type-III-secretion systems, the BID domain might similarly act as an unfoldase to prime toxins, relaxases, and Beps for translocation (Stanger et al., 2017). Future work may address the question if BID domains indeed function as chaperones prior to and after T4S. Furthermore, using multidisciplinary approaches such as X-ray crystallography and cryo-electron tomography, future efforts could pave the way for elucidating long-lasting questions regarding the T4S pathway(s) of relaxases and effectors by using BID domain-containing T4S substrates.

\section{AUTHOR CONTRIBUTIONS}

AW and CT designed the figures. AW, CT, and CD wrote the manuscript.

\section{FUNDING}

This work was supported by grant 31003A_173119 to CD from the Swiss National Science Foundation (SNSF, www.snf.ch).

Kirby, J. E., and Nekorchuk, D. M. (2002). Bartonella-associated endothelial proliferation depends on inhibition of apoptosis. Proc. Natl. Acad. Sci. USA 99, 4656-4661. doi: 10.1073/pnas.072292699

Li, Y. G., and Christie, P. J. (2018). The agrobacterium VirB/VirD4 T4SS: mechanism and architecture defined through in vivo mutagenesis and chimeric systems. Curr. Top. Microbiol. Immunol. 418, 233-260. doi: 10.1007/82_2018_94

Okujava, R., Guye, P., Lu, Y. Y., Mistl, C., Polus, F., Vayssier-Taussat, M., et al. (2014). A translocated effector required for Bartonella dissemination from derma to blood safeguards migratory host cells from damage by co-translocated effectors. PLoS Pathog. 10:e1004187. doi: 10.1371/journal.ppat.1004187

Pulliainen, A. T., Pieles, K., Brand, C. S., Hauert, B., Bohm, A., Quebatte, M., et al. (2012). Bacterial effector binds host cell adenylyl cyclase to potentiate Galphas-dependent cAMP production. Proc. Natl. Acad. Sci. USA 109, 9581-9586. doi: 10.1073/pnas.1117651109

Redzej, A., Ilangovan, A., Lang, S., Gruber, C. J., Topf, M., Zangger, K., et al. (2013). Structure of a translocation signal domain mediating conjugative transfer by type IV secretion systems. Mol. Microbiol. 89, 324-333. doi: $10.1111 / \mathrm{mmi} .12275$

Rhomberg, T. A., Truttmann, M. C., Guye, P., Ellner, Y., and Dehio, C. (2009). A translocated protein of Bartonella henselae interferes with endocytic uptake of individual bacteria and triggers uptake of large bacterial aggregates via the invasome. Cell. Microbiol. 11, 927-945. doi: 10.1111/j.1462-5822.2009.01302.x

Sadana, R., and Dessauer, C. W. (2009). Physiological roles for G proteinregulated adenylyl cyclase isoforms: insights from knockout and overexpression studies. Neurosignals 17, 5-22. doi: 10.1159/000166277

Scherer, D. C., Deburon-Connors, I., and Minnick, M. F. (1993). Characterization of Bartonella bacilliformis flagella and effect of antiflagellin antibodies on invasion of human erythrocytes. Infect. Immun. 61, 4962-4971.

Schmid, M. C., Scheidegger, F., Dehio, M., Balmelle-Devaux, N., Schulein, R., Guye, P., et al. (2006). A translocated bacterial protein protects vascular endothelial cells from apoptosis. PLoS Pathog. 2:e115. doi: 10.1371/journal. ppat.0020115

Schmid, M. C., Schulein, R., Dehio, M., Denecker, G., Carena, I., and Dehio, C. (2004). The VirB type IV secretion system of Bartonella henselae mediates 
invasion, proinflammatory activation and antiapoptotic protection of endothelial cells. Mol. Microbiol. 52, 81-92. doi: 10.1111/j.1365-2958.2003.03964.x

Schulein, R., Guye, P., Rhomberg, T. A., Schmid, M. C., Schroder, G., Vergunst, A. C., et al. (2005). A bipartite signal mediates the transfer of type IV secretion substrates of Bartonella henselae into human cells. Proc. Natl. Acad. Sci. USA 102, 856-861. doi: 10.1073/pnas.0406796102

Schulein, R., Seubert, A., Gille, C., Lanz, C., Hansmann, Y., Piemont, Y., et al. (2001). Invasion and persistent intracellular colonization of erythrocytes. A unique parasitic strategy of the emerging pathogen Bartonella. J. Exp. Med. 193, 1077-1086. doi: 10.1084/jem.193.9.1077

Selbach, M., Paul, F. E., Brandt, S., Guye, P., Daumke, O., Backert, S., et al. (2009). Host cell interactome of tyrosine-phosphorylated bacterial proteins. Cell Host Microbe 5, 397-403. doi: 10.1016/j.chom.2009.03.004

Siamer, S., and Dehio, C. (2015). New insights into the role of Bartonella effector proteins in pathogenesis. Curr. Opin. Microbiol. 23, 80-85. doi: 10.1016/j.mib.2014.11.007

Stanger, F. V., De Beer, T. A. P., Dranow, D. M., Schirmer, T., Phan, I., and Dehio, C. (2017). The BID domain of type IV secretion substrates forms a conserved four-helix bundle topped with a hook. Structure 25, 203-211. doi: $10.1016 /$ j.str.2016.10.010

Trokter, M., and Waksman, G. (2018). Translocation through the conjugative type 4 secretion system requires unfolding of its protein substrate. J. Bacteriol. 200:e00615-17. doi: 10.1128/jb.00615-17

Truttmann, M. C., Guye, P., and Dehio, C. (2011). BID-F1 and BID-F2 domains of Bartonella henselae effector protein BepF trigger together with BepC the formation of invasome structures. PLoS One 6:e25106. doi: 10.1371/journal. pone.0025106

Truttmann, M. C., Rhomberg, T. A., and Dehio, C. (2011). Combined action of the type IV secretion effector proteins BepC and BepF promotes invasome formation of Bartonella henselae on endothelial and epithelial cells. Cell. Microbiol. 13, 284-299. doi: 10.1111/j.1462-5822.2010.01535.x

Vieira-Damiani, G., Ericson, M. E., Da Silva, M. N., Gupta, K., Soares, T. B., De Almeida, A. R., et al. (2016). Bartonella henselae initial infection of mature human erythrocytes observed in real time using bacterial endogenous fluorescence. J. Trop. Dis. Public Health 4:207. doi: 10.4172/2329-891x.1000207

Wagner, A., and Dehio, C. (2019). Role of distinct type-IV-secretion systems and secreted effector sets in host adaptation by pathogenic Bartonella species. Cell. Microbiol. 21:e13004. doi: 10.1111/cmi.13004

Waksman, G. (2019). From conjugation to T4S systems in Gram-negative bacteria: a mechanistic biology perspective. EMBO Rep. 20:e47012. doi: 10.15252/embr.201847012

Wang, C., Fu, J., Wang, M., Cai, Y., Hua, X., Du, Y., et al. (2018). Bartonella quintana type IV secretion effector BepE-induced selective autophagy by conjugation with K63 polyubiquitin chain. Cell. Microbiol. 21:e12984. doi: $10.1111 / \mathrm{cmi} .12984$

Conflict of Interest Statement: The authors declare that the research was conducted in the absence of any commercial or financial relationships that could be construed as a potential conflict of interest.

Copyright (c) 2019 Wagner, Tittes and Dehio. This is an open-access article distributed under the terms of the Creative Commons Attribution License (CC BY). The use, distribution or reproduction in other forums is permitted, provided the original author(s) and the copyright owner(s) are credited and that the original publication in this journal is cited, in accordance with accepted academic practice. No use, distribution or reproduction is permitted which does not comply with these terms. 